\title{
Obtención de un Sustituto de Chocolate tipo-Pasta usando Pulpa de Carao (Cassia fistula L.)
}

\author{
Lena Morón, Yohana Caro, Rafael E. Gonzálezo y Érica P. Torres \\ Universidad de Cartagena, Piedra de Bolívar - Av Del Consulado, Calle 30 № 48-157., Colombia. \\ (e mail: Imorona@unicartagena.edu.co)
}

Recibido Mar. 18, 2015; Aceptado May. 18, 2015; Versión final Jun. 25, 2015; Publicado Dic. 2015

\begin{abstract}
Resumen
En la presente investigación se elaboró un sustituto de chocolate a base de pulpa de carao (Cassia fistula L.), formulada con el $54.78 \%(\mathrm{p} / \mathrm{p})$ de pulpa, $25.11 \%(\mathrm{p} / \mathrm{p})$ de sacarosa para la primera formulación $(\mathrm{F} 1)$ y para la segunda formulación (F2) se adicionó $0.16 \%$ (2g) de Stevia Rebaudiana Bertoni. Se evaluaron las propiedades microbiológicas, bromatológicas y sensoriales. Los resultados indicaron el uso de Stevia como endulzante modifica las propiedades bromatológicas de la pasta de carao 2 (F2), ya que se presentaron diferencias estadísticas significativas $(p<0,05)$ con respecto a la pasta de carao 1 . El contenido de humedad $(49.99 ; 58.18 \%)$, proteínas $(7.32 ; 8.59 \%)$ y grasas $(6.61 ; 6.31 \%)$ fue mayor en las pastas 1 y 2 respectivamente en comparación con la materia prima que mostró porcentajes de $39.15 \%$ para humedad, $3.82 \%$ de proteína y $4.03 \%$ de grasa, debido a la adición de agua y leche en polvo. El análisis sensorial llevado a cabo indicó la posibilidad de utilizar la pulpa de carao como sustituto de chocolate.
\end{abstract}

Palabras clave: pulpa de carao, sustituto de chocolate, propiedades físicas, evaluación sensorial

\section{Obtaining a Substitute of Paste-type Chocolate using Carao pulp (Cassia fistula L.)}

\begin{abstract}
In this research a chocolate substitute was obtained from carao pulp (Cassia fistula L.), formulated with $54.78 \%(\mathrm{w} / \mathrm{w})$ of pulp, $25.11 \%(\mathrm{w} / \mathrm{w})$ of sucrose to the first formulation ( $\mathrm{F} 1)$ and for the second formulation (F2) $0.16 \%$ (2g) of Stevia Rebaudiana Bertoni was added. Microbiological, bromatological and sensorial properties were evaluated. Results indicated that using Stevia as a sweetener modifies the bromatological properties of carao pulp 2 (F2), because significant statistical differences $(\mathrm{p}<0,05)$ were presented with respect to the carao pulp 1 . The moisture content $(49.99 ; 58.18 \%)$, protein $(7.32 ; 8.59 \%)$ and fat $(6.61$; $6.31 \%$ ) were higher in the pulp 1 and 2 respectively in comparison with the raw material which showed percentages of $39.15 \%$ moisture, $3.82 \%$ protein and $4.03 \%$ fat, due to the water and powdered milk addition. The sensorial analysis carried out indicated the possibility of using carao pulp as a substitute of chocolate.
\end{abstract}




\section{INTRODUCCIÓN}

El chocolate es un producto obtenido a partir de cacao que contiene aproximadamente $350 \mathrm{~g} / \mathrm{kg}$ de materia seca de cacao (Torres et al., 2011) que se metaboliza y digiere rápidamente por el organismo, además posee un alto contenido energético; su textura y sabor son las principales razones que favorecen su elevado consumo. Por su alto contenido de grasa saturada y colesterol (30\%) incrementa el riesgo de enfermedades cardiovasculares (Konar, 2013). Por consiguiente, es necesaria la reducción de la grasa en el chocolate; aunque es una tarea difícil, ya que la manteca de cacao juega un papel importante en la calidad y procesamiento del mismo (Tayefe, 2010).

La Norma Técnica Colombiana (NTC 792/2008) establece que los sucedáneos de chocolate para consumo directo son obtenidos por sustitución de la manteca de cacao por otras grasas vegetales en proporciones variables. Según Hoda et al., (2006) los sustitutos de cacao pueden ser preparados mediante el uso de fuentes vegetales comestibles adecuadas. La Cassia fistula Linn. (Cassia) familia Caesalpiniaceae comúnmente conocido como Amulthus podría ser utilizada como sustituto del cacao en la elaboración del chocolate debido a que es una fruta baja en grasa y azúcares; igualmente, contiene aceites volátiles, sustancias cerosas, agua, proteínas y aminoácidos esenciales como arginina, leucina, metionina, fenilalanina y triptófano (Thirumal et al., 2012). Además, es una buena fuente de minerales como Fe y Mn en comparación con otros frutos como la manzana, el albaricoque, el melocotón, la pera y la naranja (Reyhan et al., 2014).

En la actualidad, el reto de la industria de alimentos consiste en generar alternativas que sustituyan el chocolate tradicional por análogos bajos en grasa con atributos sensoriales similares al producto original, así mismo sin variaciones en las condiciones de procesamiento (Tayefe et al., 2014). Salem et al., (2012) utilizaron el polvo de la vaina de algarroba debido a sus valores nutricionales y saludables como sustituto de chocolate, mejorando los valores nutricionales, las propiedades funcionales y sensoriales del producto, siendo una herramienta de éxito para sustituir las semillas de cacao en los productos de chocolate. Por tal motivo, el objetivo del siguiente trabajo fue desarrollar un producto tipo pasta a partir de pulpa de Carao (Cassia fistula L.), y evaluar su calidad nutricional, sensorial y microbiológica; para ser utilizado como sustituto de chocolate.

\section{MATERIALES Y MÉTODOS}

\section{Materia prima}

Se utilizó como materia prima frutos de Carao (Cassia fistula L.) obtenidas del corregimiento de Santa Ana, Municipio de Cartagena - Bolívar. La Stevia (Erba dolce), la lecitina de soya (Juliao), la vainillina (Juliao) y la leche en polvo semidescremada (Coolechera) se obtuvieron en un supermercado local.

\section{Elaboración del sustituto de chocolate tipo pasta}

Las vainas de carao (Casssia fistula), se seleccionaron de acuerdo a sus condiciones físicas (sin daños mecánicos) y su estado de madurez según (Geilfus 1989; Rojas y Torres, 2012). Para la limpieza de las vainas del fruto de carao, se realizó un lavado con abundante agua con el objetivo de eliminar agentes físicos de la corteza, luego fueron secadas a temperatura ambiente. Posteriormente, la pulpa de carao fue extraída de manera artesanal evitando cualquier residuo contaminante de la vaina. En seguida la pulpa fue fermentada con $50 \%(\mathrm{p} / \mathrm{p})$ de sacarosa a $37^{\circ} \mathrm{C}$ durante 6 días. El tamaño de las partículas fue reducido mezclando el producto obtenido con los demás ingredientes propuestos por Nebesny y Zyzelewicz, (2005) (Ver tabla 1), con el fin de obtener una pasta fina y homogénea. Finalmente la preparación fue sometida a tratamiento térmico $\left(80^{\circ} \mathrm{C} / 30\right.$ minutos) obteniendo así la primera pasta de carao. Para la obtención de la segunda pasta de carao; la concentración de sacarosa fue reemplazada por un endulzante natural (Stevia Rebaudiana Bertoni), para obtener un producto más saludable.

Tabla 1: formulación propuesta por Nebesny y Zyzelewicz (2005)

\begin{tabular}{|c|c|c|}
\hline Ingredientes & $\%(\mathrm{p} / \mathrm{p})$ & Cantidad $\mathrm{g}$ \\
\hline Pulpa & 54,78 & 440,3 \\
\hline Leche en polvo & 20 & 160,75 \\
\hline Sacarosa & 25,11 & 201,82 \\
\hline Lecitina & 0,1 & 0,803 \\
\hline Vanillina & 0,001 & 0,0803 \\
\hline
\end{tabular}




\section{Análisis microbiológicos}

Se tomaron $11 \mathrm{~g}$ de las muestras y se adicionaron en $99 \mathrm{~mL}$ de agua de peptona, posteriormente se realizaron diluciones seriadas consecutivas y finalmente se determinó la concentración bacteriana de: mohos y levaduras, aerobios mesófilos y coliformes fecales utilizando agar sabouraud, agar plate count y caldo bilis verde brillante respectivamente, según la Norma Técnica Colombiana 792 (NTC 792) tercera actualización; Los análisis microbiológicos fueron llevados a cabo tanto a la materia prima como al producto terminado.

\section{Análisis bromatológicos}

Los análisis de humedad, proteínas, grasas, carbohidratos, azúcar reductor y cenizas fueron llevados a cabo conforme lo establecen los métodos de la AOAC, (2003). El pH fue determinado con un potenciómetro digital (Beckman, USA). Todos los análisis fueron realizados por triplicado, tanto para la materia prima como para las pastas de carao 1 y 2.

\section{Evaluación sensorial}

La evaluación sensorial (color, olor, sabor y aceptación general) de las pastas de chocolate, se llevó a cabo con un panel de 30 jueces (estudiantes universitarios escogidos al azar con edades entre 18 y 23 años) familiarizados con el producto, dividido en tres secciones de 10 panelistas. Este análisis se basó en una escala hedónica de 9 puntos (donde 1 es me gusta muchísimo y 9 es me disgusta muchísimo). Las muestras se presentaron en recipientes plásticos libres de olores y bajo las mismas condiciones para todos los jueces. Se utilizó agua para el lavado de la boca de los panelistas entre muestras.

\section{Análisis estadístico}

Los resultados de los análisis bromatológicos y sensoriales fueron analizados mediante análisis normal de varianza (ANOVA un factor) con el fin de establecer diferencias estadísticas significativas $(p<0,05)$ entre las pastas de carao analizadas. Para tal fin se utilizó el programa de computo SPSS (ver 13,0 para Windows) empleando la prueba DMS (diferencias mínimas significativas).

\section{RESULTADOS Y DISCUSIÓN}

\section{Análisis microbiológicos}

Una de las formas de establecer la calidad de un producto alimenticio es a través de la enumeración de microorganismos de interés alimentario (Vásquez, 2003), por ende, la determinación de microorganismos aerobios mesófilos y hongos en la pasta de chocolate tiene gran relevancia en la aceptación final del producto alimenticio por parte del consumidor. En ambas pastas de carao ( $F 1$ y $F 2$ ) se presentaron concentraciones microbianas menores a $10 \mathrm{UFC} / \mathrm{g}$ las cuales se encuentran por debajo del límite exigido por la legislación Colombiana (NTC,792:2008), que establece para chocolates y sucedáneos los límites máximos permitidos para estos microorganismos en $10 \mathrm{UFC} / \mathrm{g}$. Con respecto al análisis de Coliformes totales y E. coli se pudo apreciar que no existe ningún tipo de contaminación por estas bacterias, ya que se obtuvieron valores menores a 3 bacterias por gramo del producto alimenticio; por ende, tanto la materia prima como las pastas de carao sustitutas de chocolate 1 y 2 presentan una calidad microbiológica aceptable para el consumo. Resultados similares fueron reportados por Soto y Caballero (2011) para chocolates suplementados con hierro hemo, estos resultados indican que la materia prima y las condiciones higiénico-sanitarias de elaboración del chocolate fueron idóneas.

\section{Análisis bromatológicos}

Los resultados de los análisis bromatológicos se presentan en la tabla 2. La humedad de la materia prima fue de $39,15 \%$ apreciándose diferencias estadísticas significativas $(P<0,05)$ entre las pastas de carao sustitutos de chocolate, con humedades de $49,99 \%$ y $58,18 \%$ para la pasta 1 y pasta 2 respectivamente, este incremento proviene de la adición de agua durante la elaboración de la pasta de carao. Es importante destacar que la adición de agua no conllevó al crecimiento microbiano, ya que no se apreció aumento en el número de microorganismos en las formulaciones ni en la materia prima; por tal motivo, es factible que el carao tenga sustancias bacteriostáticas naturales que afecten el crecimiento microbiano, debido a que muchos alimentos contienen compuestos naturales con actividad antimicrobiana, que en estado natural pueden desempeñar el papel de prolongadores de vida útil del propio alimento (Rodríguez, 2011). 
En cuanto al contenido de grasa, no se observaron diferencias estadísticas significativas $(P<0,05)$ entre las pastas de carao, para la pasta 1 se obtuvo 6,61\% mientras que en la pasta 2 el valor fue de $6,31 \%$ por el contrario en la materia prima se apreció una disminución (4,03\%), este valor concuerda con el reportado por Salem y Fahad, (2012) quienes obtuvieron concentraciones de grasa de 4,80\% en algarrobo en polvo; el cual fue utilizado como sustituto de la semilla de cacao para la elaboración de chocolate con leche. Estos autores reportaron que cuando se sustituía completamente el cacao por el algarrobo se obtenía un nivel de grasa en la formulación final cercana al $24 \%$, siendo este valor superior al reportado en esta investigación para las pastas de carao sustitutas de chocolate 1 y 2 . Aunque ambos porcentajes de grasas son bajos comparándolos con el chocolate tradicional que contienen aproximadamente un $70 \%$ de grasa en su fase continua (Ohene et al., 2008).

Con respecto al contenido de proteínas de ambas pastas (F1 y F2) y de la materia prima, se observó una variación significativa $(P<0.05)$. Mientras que en la materia prima obtuvo $3,82 \%$, en las pastas 1 y 2 se obtuvieron concentraciones de $7,32 \%$ y $8,59 \%$ respectivamente, este aumento, se debe a la adición de leche en polvo en las formulaciones. Shah et al., (2010), evidenciaron que al adicionar proteína de suero de leche, el contenido de proteína en el chocolate aumenta paulatinamente. Resultados similares fueron reportados por Salem y Fahad, (2012) en la elaboración de chocolate, donde el contenido proteico se incrementó como consecuencia del aumento significativo de las proporciones de polvo de algarrobo desde $7.91 \%$ hasta $8.47 \%$ de proteína.

El contenido de carbohidratos en la materia prima utilizada como sustituto fue de $48,14 \%$, este porcentaje es menor en comparación con otros sustitutos como el algarrobo que contiene $51,76 \%$ de carbohidratos (Salem y Fahad, 2012). Las pastas de carao arrojaron concentraciones de carbohidratos de $32,84 \%$ y $24,08 \%$ para las pastas 1 y 2 respectivamente. Este comportamiento es normal si se tiene en cuenta que la pasta 2 debe presentar una menor concentración, debido a la adición de Stevia Rebaudiana en sustitución de sacarosa. Las concentraciones de carbohidratos reportadas para pastas 1 y 2 son menores a las reportadas en chocolates con leche tradicionales como las encontradas por Chan et al., (1994) donde la cantidad de carbohidrato en su formulación de chocolate con leche fue de $56,90 \%$. El porcentaje de azúcares reductores de las formulaciones y la materia prima no presentaron diferencias estadísticamente significativas $(\mathrm{P}<0,05)$, este comportamiento puede ser atribuido a la fermentación llevada a cabo en la obtención de las pastas. Biehl et al., (1989), reportaron concentraciones de azúcares menores en la pulpa cuando se fermentaban combinaciones de cacao amazónico y amelonados, con el método de caja. Por el contrario, en los niveles de cenizas si presentaron diferencias significativas $(P<0,05)$ entre las muestras analizadas, ya que la materia prima tuvo una mayor concentración (4,85\%) en comparación con la pasta 1 $(3,22 \%)$ y pasta $2(2,81 \%)$, esta diferencia puede ser ocasionada por la dilución de los minerales presentes en la pulpa por la adición de agua durante el proceso de elaboración de la pasta.

Tabla 2: Características bromatológicas del fruto y las pastas de carao. Filas sin ninguna letra en común presentaron diferencias estadísticas a un nivel de confianza $(P<0,05)$.

\begin{tabular}{|c|c|c|c|c|c|c|}
\hline Muestras & Humedad & Grasas & Proteínas & Carbohidratos & Azúcar Reductor & Cenizas \\
\hline Materia Prima & $39,15 \pm 0,60^{\mathrm{a}}$ & $4,03 \pm 0,18^{\mathrm{a}}$ & $3,82 \pm 0,12^{\mathrm{a}}$ & $48,14 \pm 1,30^{\mathrm{a}}$ & $1,39 \pm 0,05^{\mathrm{a}}$ & $4,85 \pm 1,05^{\mathrm{a}}$ \\
\hline Pasta de Carao 1 & $49,99 \pm 0,86_{\mathrm{b}}$ & $6,61 \pm 0,21^{\mathrm{b}}$ & $7,32 \pm 0,31^{\mathrm{b}}$ & $32,84 \pm 0,36^{\mathrm{b}}$ & $1,43 \pm 0,00^{\mathrm{a}}$ & $3,22 \pm 1,09^{\mathrm{b}}$ \\
\hline Pasta de Carao 2 & $58,18 \pm 0,21^{\mathrm{c}}$ & $6,31 \pm 0,11^{\mathrm{b}}$ & $8,59 \pm 0,15^{\mathrm{c}}$ & $24,08 \pm 0,12^{\mathrm{c}}$ & $1,24 \pm 0,01^{\mathrm{a}}$ & $2,81 \pm 0,06^{\mathrm{c}}$ \\
\hline
\end{tabular}

\section{Evaluación sensorial}

Para la evaluación sensorial se utilizó una escala hedónica de 9 puntos donde se evaluaron atributos tales como color, olor, sabor y aceptación general para cada sustituto de pasta de chocolate. Los resultados obtenidos se presentan en la tabla 3, donde se puede observar que no se encontraron diferencias significativas $(P<0,5)$ entre ambas pastas de carao para cada uno de los atributos sensoriales evaluados, estos resultados fueron similares a los encontrados por Salem y Fahad (2012), quienes utilizaron el polvo de algarrobo como sustituto de chocolate con leche. El atributo sensorial de mayor puntuación promedio se encontró en el sabor para la pasta $2(4,76)$, lo cual puede ser atribuido al uso de Stevia como endulzante, lo que quiere decir que no afectó el dulzor de la pasta de chocolate, caso similar resultó en el estudio hecho por Martins et al., (2009) quienes utilizaron Sucralosa y Stevia como edulcorantes en la elaboración de chocolate bajo en calorías, los cuales tuvieron un aumento en el sabor dulce del chocolate, siendo el de mayor regusto el que contenía Stevia; por otro lado la menor puntuación encontrada fue para el color en la pasta $1(3,04)$ presentando diferencias significativas con la pasta de carao 2, en cuanto a la aceptación general no se mostraron diferencias significativas $(P<0,5)$ teniendo un valor promedio de aceptación de 4,56 lo que indica la posibilidad de utilizar la pulpa de carao como sustituto de chocolate debido a su nivel de aceptación. 
Tabla 3: Valores promedios de la puntuación obtenida en el análisis sensorial. Columnas sin ninguna letra en común presentaron diferencias estadísticas significativas a un nivel de confianza de del $95 \%$.

\begin{tabular}{|c|c|c|c|c|}
\hline Muestra & Color & Olor & Sabor & Aceptación General \\
\hline Pasta de Carao 1 & $3,04 \pm 1,670^{\mathrm{a}}$ & $4,56 \pm 2,599^{\mathrm{a}}$ & $4,24 \pm 2,471^{\mathrm{a}}$ & $4,68 \pm 2,512^{\mathrm{a}}$ \\
\hline Pasta de Carao 2 & $3,52 \pm 1,828^{\mathrm{a}}$ & $4,72 \pm 2,747^{\mathrm{a}}$ & $4,76 \pm 2,570^{\mathrm{a}}$ & $4,44 \pm 2,274^{\mathrm{a}}$ \\
\hline
\end{tabular}

\section{CONCLUSIONES}

La pulpa y la pasta de carao presentan propiedades microbiológicas aceptables para ser consumidos sin ocasionar riesgo para la salud de las personas. Además, las pastas de carao presentan menores contenidos de grasa y carbohidratos totales en comparación al chocolate. La adición de Stevia contribuye a la disminución de carbohidratos en la pasta de carao (F2). Estos resultados sugieren que la pasta de carao se puede utilizar como sustituto de chocolate para reducir el contenido de grasa y azúcar que aportan los chocolates tradicionales, de la misma manera la aceptación general de la pasta de carao en el análisis sensorial llevado a cabo indica esta posibilidad.

\section{REFERENCIAS}

Biehl, B. y otros tres autores, Chemical and physical changes in the pulp during ripening and post-harvest storage of Cocoa Pads, J. Sci. food. agric. 48(2),189-208 (1989)

Chan, W. y otros tres autores, Miscellaneous foods. Supplement to McCane and Widdowson's the composition of foods. London: RSC/MAFF. (1994)

Geilfus, F., El árbol al servicio del agricultor: Manual de agroforestería para el desarrollo rural. Santo Domingo, DO, Editorial Sto. Domingo, 508 - 509 (1989)

Hoda, H. y otros tres autores, Cocoa substitute: Evaluation of sensory qualities and flavour stability, Eur Food Res Technol, 223(1), 125-131 (2006)

Konar, N., Influence of conching temperature and some bulk sweeteners on physical and rheological properties of prebiotic milk chocolate containing inulin, Eur Food Res Technol, 236,135-143 (2013)

Martins, L. y otros tres autores, Sensory profile, acceptability, and their relationship for diabetic/reduced calorie chocolates, Food Qual Pref, 20, 138-143 (2009)

Nebesny E. y D. Zyzelewicz, Effect of lecithin concentration on properties of sucrose-free chocolate masses sweetened with isomalt, Eur Food Res Technol. 220, 131-135. (2005)

NTC 792-04-09, tercera actualización, Norma Técnica Colombiana sobre chocolate y sus sucedáneos para consumo directo, 03-26, Bogotá-Colombia (2008)

Ohene A.E., A. Patersona y M. Fowler, Factors influencing rheological and textural qualities in chocolate a review. Tren Food Sci Technol, 18, 290-298 (2008)

Reyhan. A. y otros seis autores, Mineral composition of pods and seeds of wild and grafted carob (Ceratonia siliqua L.) fruits. Sci Horticul, 167, 149-152 (2014)

Rodríguez S. E. Uso de Agentes Antimicrobianos Naturales en la Conservación de Frutas y Hortalizas, Ra Xim, 7 (1), 153-170 (2011)

Rojas, F. y G. Torres, Árboles del Valle Central de Costa Rica: reproducción Caña Fístula (Cassia fistula L.) Rev For Mes Kurú (Costa Rica), 9, 13 -23 (2012)

Salem E.M. Y A.O. Fahad, Substituting of Cacao by Carob Pod Powder In Milk Chocolate Manufacturing, Aus J Bas Appl Sci, 6(3), 572-578 (2012)

Shah, A.B., G. P. Jones y T. Vasiljevic, Sucrose-free chocolate sweetened with Stevia rebaudiana extract and containing different bulking agents - effects on physicochemical and sensory properties, Int J Food Sci Technol, 45, 1426-1435 (2010) 
Soto M. A. y P.L. Caballero, Adición de hierro hemo, proveniente de hemoglobina bovina a un chocolate de consumo directo, Rev Fac Cien Bás, 9(1), 21-31 (2011)

Tayefe A. N. y otros cinco autores, Development of reduced-fat and reduced-energy dark chocolate using collagen hydrolysate as cocoa butter replacement agent, J Food Nut Res 53(1), 13-21 (2014)

Thirumal. M., S. Surya y G. Kishore, Cassia fistula Linn - Pharmacognostical, Phytochemical and Pharmacological Review, Crit Rev Phar Sci, 1(1), 48-69 (2012)

Torres M.M. y otros tres autores, Dark chocolate acceptability: influence of cocoa origin and processing conditions. J Sci Food Agric, 92, 404-411 (2011)

Vásquez, G. La contaminación de los alimentos, un problema por resolver, Salud UIS, 35(1), 48-57 (2003) 